\title{
Berberine inhibits the proliferation, invasion and migration of endometrial stromal cells by downregulating miR-429
}

\author{
YONGJUAN GU ${ }^{1}$ and ZHIGANG ZHOU ${ }^{2}$ \\ ${ }^{1}$ Department of Obstetrics and Gynecology, Jianhu Hospital Affiliated to Nantong University, \\ Yancheng, Jiangsu 224700; ${ }^{2}$ Institution of Traditional Chinese Medicine, Jiangxi University of \\ Traditional Chinese Medicine, Nanchang, Jiangxi 330004, P.R. China
}

Received May 15, 2020; Accepted August 20, 2020

DOI: $10.3892 / \mathrm{mmr} .2021 .12055$

\begin{abstract}
Endometriosis (EM) is a common gynecological disease, and its pathological process is accompanied by the migration and proliferation of uterine cells. Berberine (BBR) has been shown to exhibit antitumor activity; however, the effects of BBR on EM have seldom been reported to date. The expression of microRNA (miR)-429 is upregulated in EM and miR-429 can be used as a target for drug regulation of cancer cells. Whether BBR plays a regulatory role in EM by targeting miR-429 has not been reported. Thus, the aim of the present study was to determine the effects of BBR on EM cells. The survival rate of immortalized human endometrial stromal cells (HESCs) was determined using a Cell Counting Kit-8 assay. A colony formation assay was used to detect the rate of cell proliferation. The expression levels of proliferation-related proteins, including proliferation marker protein $\mathrm{Ki}-67$ (Ki-67) and proliferating cell nuclear antigen (PCNA), were detected by reverse transcription-quantitative PCR (RT-qPCR) and western blotting. Wound healing and Transwell assays were performed to detect cell migration and invasion, and western blotting was used to detect the expression of the migration- and invasion-related proteins, including matrix metalloproteinase (MMP)2, MMP4 and MMP9. The expression of miR-429 was detected by RT-qPCR following its overexpression via cell transfection. The results revealed that treatment with $80 \mu \mathrm{M}$ BBR significantly inhibited cell proliferation and colony formation, and inhibited the expression of Ki-67 and PCNA proteins in HESCs. BBR inhibited cell invasion and migration, as well as the expression of MMP2, MMP4 and MMP9. In this process, it was found that the expression of miR-429 decreased following treatment of the cells with BBR, whereas
\end{abstract}

Correspondence to: Professor Zhigang Zhou, Institution of Traditional Chinese Medicine, Jiangxi University of Traditional Chinese Medicine, 1688 Meiling Avenue, Wanli, Nanchang, Jiangxi 330004, P.R. China

E-mail: zhouzhigang128@163.com

Key words: berberine, microRNA-429, endometriosis, proliferation, invasion, migration the inhibitory effects of BBR on cell proliferation, invasion and migration were suppressed following the overexpression of miR-429. Overall, the findings of the present study indicated that BBR inhibited the proliferation, invasion and migration of HESCs by downregulating the expression of miR-429.

\section{Introduction}

Endometriosis (EM) is a common gynecological disease, induced by the presence of an active endometrium on the lateral side of the uterine cavity (1). The pathological processes of EM include periodic bleeding, the migration of uterine cells and their attachment to other organs (2). Although EM is a benign lesion, it exhibits biological behavior similar to that of a malignant tumor, including invasion, distant metastasis and spread (3-5). Therefore, the early treatment and prevention of EM are crucial.

Berberine (BBR) is widely available in Rhizoma coptidis, Phellodendron amurense and other plants, and is a member of a class of isoquinoline alkaloids widely used in clinical practice against pathogenic microorganisms, to reduce blood sugar levels, to regulate blood lipid levels, and to protect the heart and fight inflammation (6). BBR has been reported to exert inhibitory effects on various types of tumors (7-9). BBR has been demonstrated to inhibit the growth and metastasis of endometrial cancer via the microRNA (miRNA/miR)-101/cyclooxygenase-2 (COX-2)/prostaglandin E2 signaling pathway, indicating that BBR is a potential anticancer drug for the treatment of endometrial cancer (10). In addition, BBR has been reported to exert an antitumor effect by inhibiting the proliferation and inducing the apoptosis of ovarian cancer cells (11). Therefore, it was hypothesized that BBR may exert a regulatory effect on the proliferation, invasion and migration of endometrial stromal cells in EM.

Studies have revealed that the expression of miR-429 is abnormal in a variety of cancer types. It has been reported that regulating the expression of miR-429 can regulate the proliferation, invasion and apoptosis of tumor cells (12-14). Therefore indicating that miR-429 can be used as a potential target for the regulation of cancer cells in drug therapy. The expression of miR-429 in colon cancer tissues was previously revealed to be significantly upregulated. However, following the administration of BBR, the expression of 
miR-429 in colon cancer tissues was shown to be markedly decreased, indicating that BBR may be a potential therapeutic drug for colon cancer and that miR-429 may be a target of BBR (15). In addition, it has been reported that the expression of miR-429 in the tissues of patients with EM is upregulated (16). Therefore, the present study examined the effects of BBR on the proliferation, invasion and migration of endometrial stromal cells in EM, and explored the potential underlying mechanisms involved, so as to provide a theoretical basis for BBR treatment of EM.

\section{Materials and methods}

Cells and cell culture. Immortalized human endometrial stromal cells (HESCs; CRL-2615) were purchased from The Cell Bank of Type Culture Collection of the Chinese Academy of Science, and incubated in DMEM (Gibco; Thermo Fisher Scientific, Inc.) supplemented with 10\% FBS (Gibco; Thermo Fisher Scientific, Inc.) at $37^{\circ} \mathrm{C}$ with $5 \% \mathrm{CO}_{2}$.

Reagent. BBR (cat. no. B3251-10G; Sigma-Aldrich; Merck $\mathrm{KGaA})$ was dissolved in pure methanol (100\%) and then diluted in PBS to obtain the desired concentration $(20,40,60$, $80 \mu \mathrm{M})$ before being added to the cells. The final methanol concentration was $<0.1 \%$. BBR at different concentrations $(20,40,60$ and $80 \mu \mathrm{M})$ was added to the cells, and they were incubated for $24 \mathrm{~h}$. Cells in the control group were treated with PBS at the same dose. Moreover, $80 \mu \mathrm{M}$ BBR was added to the cells at 24,48 and $72 \mathrm{~h}$ to select the most ideal conditions for subsequent experiments (10).

Cell transfection. One day prior to transfection, cells $\left(5 \times 10^{4}\right.$ cells/well $)$ were seeded into six-well plates and cultured at $37^{\circ} \mathrm{C}$ in an atmosphere containing $5 \% \mathrm{CO}_{2}$. Cells were pretreated with BBR for $2 \mathrm{~h}$ and then transfected with miR-429 mimic or negative control (mimic NC), which were purchased from Guangzhou RiboBio Co., Ltd. All transfections were performed at a concentration of $20 \mathrm{nM}$ miR-429 mimics and mimic NC using Lipofectamine ${ }^{\circledR} 2000$ (Thermo Fisher Scientific, Inc.), according to the manufacturer's instructions. The sequences of miRNAs used in the present study were as follows: miR-429 mimic, sense 5'-UAAUACUGUCUG GUAAUGCCGU-3' and antisense 5'-GGCAUUACCAGA CAGUAUUAUU-3'; NC, sense 5'-UUCUCCGAACGUGUC ACGUTT-3' and antisense 5'-ACGUGACACGUUCGGAGA ATT-3'. Follow-up experiments were performed $48 \mathrm{~h}$ after cell transfection. miR-429 was overexpressed using transfection of miR-429 mimics, and cells were then divided into the following groups: i) Control; ii) BBR; iii) $\mathrm{BBR}+$ mimic-NC (BBR was administered after transfection with empty vector); and iv) BBR + miR-429 mimic groups (BBR was administered after miR-429 overexpression).

Cell Counting Kit-8 (CCK-8) assay. Cells were seeded into 96-well plates at a density of $1 \times 10^{4}$ cells/well. Following treatment with BBR, $10 \mu$ l CCK-8 solution (Dojindo Molecular Technologies, Inc.) was added to each well and the cells were incubated at $37^{\circ} \mathrm{C}$ for $4 \mathrm{~h}$, according to the manufacturer's instructions. The absorbance was measured at $450 \mathrm{~nm}$ using a VersaMax $^{\mathrm{TM}}$ microplate reader.
Edu staining. Cells were transferred into $96-$ well plates at a density of $1 \times 10^{4}$ cells/well and cultured at $37^{\circ} \mathrm{C}$ overnight. After the corresponding treatment, cells were incubated with 5'-Ethynl-2'deoxyuridine (Edu; $10 \mu \mathrm{M})$ for $4 \mathrm{~h}$, and $50 \mu \mathrm{l}$ cell fixation solution (PBS containing 4\% paraformaldehyde) was added to the cells at room temperature for $30 \mathrm{~min}$. Then, $100 \mu$ l penetrant (PBS containing $0.5 \%$ Triton X-100) was added to each well for cell permeabilization for 10 min. The Click-iT ${ }^{\mathrm{TM}}$ Edu Alexa Fluor ${ }^{\mathrm{TM}} 488$ imaging kit (Thermo Fisher Scientific, Inc.) was used according to the manufacturer's instructions. Images were captured using a light microscope (magnification, x200; Nikon Eclipse Ti-S; Nikon Corporation).

Colony formation assay. Cells were seeded in 6-well plates at a density of $1 \times 10^{6}$ cells/well and cultured at $37^{\circ} \mathrm{C}$ for $\sim 21$ days until visible colonies appeared. The cells were fixed with pure methanol for $15 \mathrm{~min}$ at room temperature and stained with $0.1 \%(\mathrm{w} / \mathrm{v})$ crystal violet for $20 \mathrm{~min}$ at room temperature. The images of colonies growing in the plates were taken at day 21 . The cells were counted under a light contrast microscope (magnification, x100; BX51; Olympus Corporation) using Image-Pro Plus 5.0 software (Media Cybernetics, Inc.). The experiments were repeated at least 3 times.

Reverse transcription-quantitative PCR (RT-qPCR). Total RNA was extracted from the cells using RNAzol $^{\circledR}$ RT (Sigma-Aldrich; Merck KGaA), following the manufacturer's protocols. The concentration and purity of the RNA were measured using a Nanodrop ${ }^{\mathrm{TM}} 2000$ spectrophotometer (Thermo Fisher Scientific, Inc.). cDNA was synthesized using a RevertAid First Strand cDNA Synthesis kit (cat. no. K1622; Thermo Fisher Scientific, Inc.) at $\sim 65^{\circ} \mathrm{C}$ for 10 min. FastStart ${ }^{\mathrm{TM}}$ Universal SYBR-Green Master (Rox) (Roche Diagnostics) was used for quantitative PCR on a StepOnePlus ${ }^{\mathrm{TM}}$ Real-Time PCR System (Thermo Fisher Scientific, Inc.), according to the manufacturer's protocols. The amplification conditions were as follows: $95^{\circ} \mathrm{C}$ for $10 \mathrm{~min}$, followed by 40 cycles of $95^{\circ} \mathrm{C}$ for $10 \mathrm{sec}$ and $60^{\circ} \mathrm{C}$ for $60 \mathrm{sec}$. Primer sequences used in PCR were obtained from GenScript. RNA expression was normalized to the expression of U6. Primer sequences used for qPCR were as follows: miR-429 forward, 5'-ACCTCGCCACCGCCTCCCATTGTC CCGTCG-3' and reverse, 5'-TGCCAGGCCCGGGTGGGT GTGAACCGGCTTC-3'; U6 forward, 5'-CTCGCTTCGGCA GCACA-3' and reverse, 5'-ACGCTTCACGAATTTGC-3'; proliferation marker protein $\mathrm{Ki}-67$ (Ki-67) forward, 5'-AAT TCAGACTCCATGTGCCTGAG-3' and reverse, 5'-CTTGAC ACACACATTGTCCTCAGC-3'; proliferating cell nuclear antigen (PCNA) forward, 5'-CACCTTAGCACTAGTATT CGAAGCAC-3' and reverse, 5'-CACCCGACGGCATCT TTATTAC-3'; and GAPDH forward, 5'-GGAGCGAGATCC CTCCAAAAT-3' and reverse, 5'-GGCTGTTGTCATACT TCTCATGG-3'. The experiments were repeated at least 3 times. The relative expression of target genes was calculated using the $2^{-\Delta \Delta C q}$ method (17).

Western blotting. Protein was extracted from the cells with RIPA lysis buffer (Beyotime Institute of Biotechnology). Total protein was quantified using a BCA protein assay 
kit. Following extraction of the total protein from the cells, $10 \%$ SDS-PAGE was used to separate the proteins $(30 \mu \mathrm{g})$, and then separated proteins were transferred to PVDF membranes. Membranes were blocked with 5\% skimmed milk for $1 \mathrm{~h}$ at room temperature, and incubated with the following primary antibodies overnight at $4^{\circ} \mathrm{C}$ : Anti-Ki-67 (1:1,000; cat. no. ab245113; Abcam), anti-PCNA (1:1,000; cat. no. ab92552; Abcam), anti-matrix metalloproteinase (MMP)2 (1:1,000; cat. no. ab92536; Abcam), anti-MMP4 (1:1,000; cat. no. ab232865; Abcam), anti-MMP9 (1:1,000; cat. no. ab76003; Abcam) and anti-GAPDH $(1: 1,000$; cat. no. ab8245; Abcam). Following which, membranes were incubated with a horseradish peroxidase-conjugated secondary antibody (1:5,000; cat. no. ab6721; Abcam) for $2 \mathrm{~h}$ at room temperature. Proteins were detected by the ECL ${ }^{\mathrm{TM}}$ Western Blotting Analysis System (GE Healthcare). The experiments were repeated at least 3 times. Protein expression levels were semi-quantified using ImageJ software (version 146; National Institutes of Health).

Wound healing assay. Cells at a density of $1 \times 10^{6}$ cells/well were allowed to grow until $90 \%$ confluency, and a gap was then created using a $200-\mu 1$ pipette tip. The cells were then cultured with fresh DMEM medium. The distance of wound closure was measured at 0 and $24 \mathrm{~h}$ with a light contrast microscope (magnification, x100; BX51; Olympus Corporation) and cells at the edges of the scratch were monitored. The percentage wound closure was determined using the following equation: $\left(A_{0}-A_{t}\right) / A_{0} \times 100$, where $A_{0}$ is the wound area at the $h$ of the wound generation, and $A_{t}$ is the wound area at the $h$ of observation. The experiments were repeated at least 3 times.

Transwell assay. For the cell invasion assay, 24-well Transwell plates (Corning, Inc.) with $8-\mu \mathrm{m}$ pore inserts coated with Matrigel (BD Biosciences) were used. Plates had been precoated with Matrigel at $37^{\circ} \mathrm{C}$ for $30 \mathrm{~min}$. Cells $\left(\sim 2 \times 10^{4}\right)$ were seeded into the Matrigel matrix-coated (Corning, Inc.) upper chambers with cell culture fluid, and DMEM supplemented with $10 \%$ FBS was added to the lower chambers. After $24 \mathrm{~h}$, the Matrigel and cells were removed using a cotton-tipped swab. The filters were fixed in $4 \%$ formaldehyde for $15 \mathrm{~min}$ at $25^{\circ} \mathrm{C}$ and stained with $0.1 \%$ crystal violet solution for $30 \mathrm{~min}$ at room temperature. Cells in five random fields were observed under a light contrast microscope (magnification, x100; BX51; Olympus Corporation). The experiments were repeated at least 3 times.

Statistical analysis. Data are presented as the mean \pm standard deviation. SPSS 17.0 statistical software (SPSS, Inc.) was used for all statistical analyses. Comparisons between groups were performed using one-way analysis of variance followed by Tukey's post hoc test. $\mathrm{P}<0.05$ was considered to indicate a statistically significant difference. All experiments were repeated at least 3 times.

\section{Results}

BBR inhibits the viability of HESCs. A CCK-8 assay was performed to determine the effects of various concentrations of $\operatorname{BBR}(20,40,60$ and $80 \mu \mathrm{M})$ on the survival rate of HESCs. It was found that treatment with $80 \mu \mathrm{M}$ BBR significantly inhibited the viability of the cells; thus, the concentration of $80 \mu \mathrm{M}$ BBR was selected for use in the following experiments (Fig. 1A). Subsequently, the HESCs were treated with $80 \mu \mathrm{M}$ BBR for 24, 48 and $72 \mathrm{~h}$. Compared with the control group, the cell survival rate decreased following treatment with BBR (Fig. 1B). The colony formation assay then showed that there was a notable decrease in cell proliferation following BBR treatment compared with the control group (Fig. 1C). Edu staining was performed to detect cell proliferation, and the results demonstrated that cell proliferation in the BBR group notably decreased compared with the control (Fig. 1D). In addition, the expression levels of the proliferation-related proteins, Ki-67 and PCNA, were also detected, and it was found that the expression levels of $\mathrm{Ki}-67$ and PCNA proteins significantly decreased following BBR treatment compared with the control group (Fig. 1E and F).

$B B R$ inhibits the migration and invasion of HESCs. Wound healing and Transwell assays revealed that the cells treated with BBR exhibited a significant decrease in cell migratory (Fig. 2A and B) and invasive (Fig. 2C and D) activity compared with the control group, which was also accompanied by a decrease in the expression levels of the invasion- and migration-related proteins, MMP2 and MMP9 (Fig. 2E). The experimental results revealed that BBR significantly inhibited the migration and invasion of endometrial stromal cells.

$B B R$ inhibits the proliferation, migration and invasion of HESCs by downregulating miR-429. During the experiment, it was found that the expression of miR-429 in the BBR-treated cells significantly decreased compared with the control group (Fig. 3A). Therefore, it was hypothesized that miR-429 may be a target of BBR in the treatment of EM. To provide evidence for this hypothesis, miR-429 was overexpressed using transfection of miR-429 mimics, and the transfection efficiency was detected by RT-qPCR (Fig. 3B). The results of the CCK- 8 and colony formation assay revealed that compared with the BBR + mimic-NC group, the overexpression of miR-429 in the BBR + miR-429 mimic group reversed the inhibitory effects of BBR on the survival rate and proliferation of HESCs (Fig. 4A and B). The results of the EdU staining showed that the overexpression of miR-429 reversed the inhibitory effect of BBR on the proliferation of HESCs compared with BBR + mimic-NC (Fig. 4C). The protein expression levels of $\mathrm{Ki}-67$ and PCNA in the BBR + miR-429 mimic group were also significantly increased compared with the BBR + mimic-NC group (Fig. 4D). The results of RT-qPCR (Fig. 4E) were consistent with those obtained for western blotting in Fig. 4D.

Wound healing and Transwell assays revealed that the overexpression of miR-429 in the BBR + miR-429 mimic group reversed the inhibitory effects of $\mathrm{BBR}$ on the migration (Fig. 5A and B) and invasion (Fig. 5C and D) of HESCs, which was accompanied by an increase in $\mathrm{Ki}-67$ and PCNA protein expression (Fig. 5E). These findings indicated that BBR inhibited the proliferation, invasion and migration of HESCs by downregulating miR-429. 
A

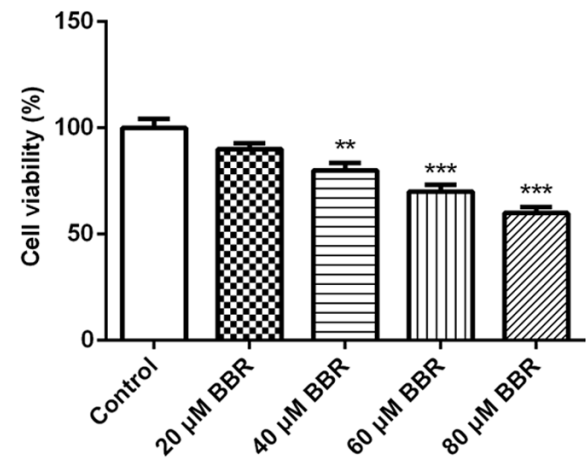

B

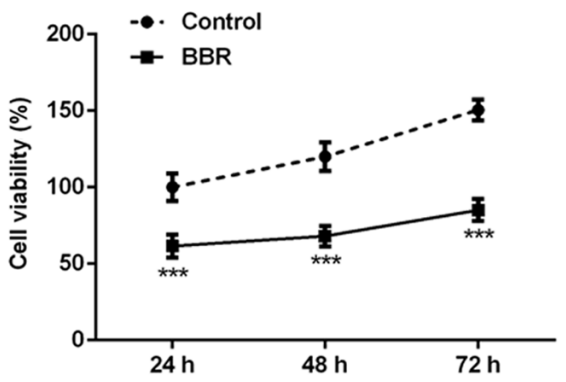

C

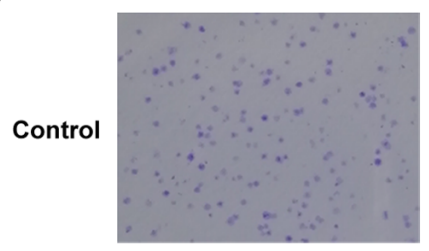

BBR

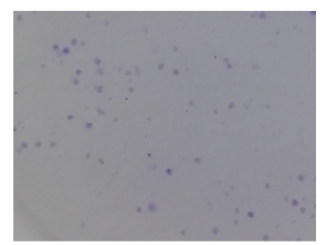

D

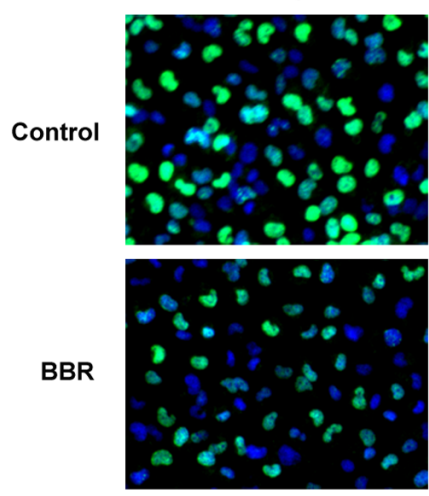

Ki-67
DAPI
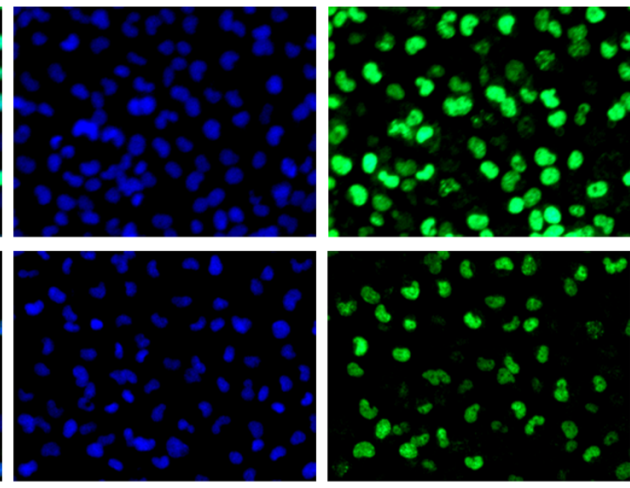

PCNA
E

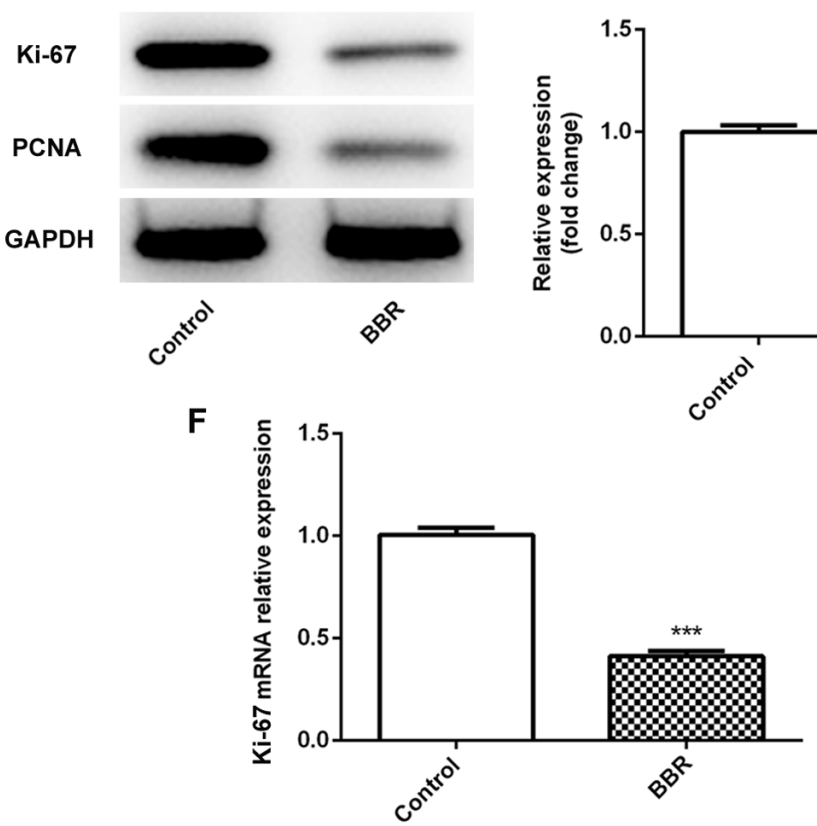

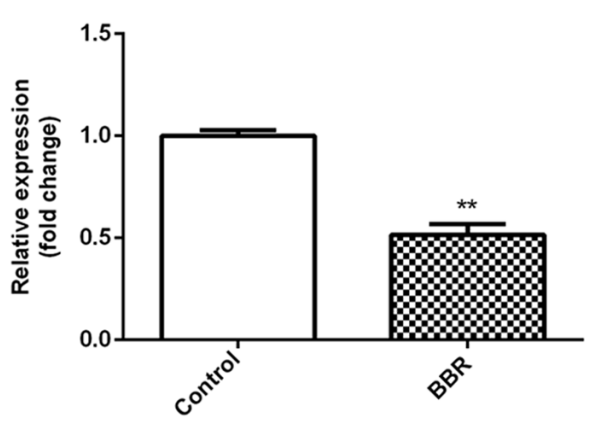

Figure 1. BBR inhibits the proliferation of human endometrial stromal cells. (A and B) A Cell Counting Kit-8 assay was used to detect cell viability following BBR treatment. (C) Cell proliferation was detected by a colony formation assay. (D) EdU staining assay was used to detect cell proliferation. (E) The expression levels of proteins Ki-67 and PCNA were detected by western blotting. (F) The expression levels of Ki-67 and PCNA mRNA were detected by reverse transcription-quantitative PCR. ${ }^{* *} \mathrm{P}<0.01,{ }^{* * *} \mathrm{P}<0.001$ vs. Control. BBR, berberine; Ki-67, proliferation marker protein Ki-67; PCNA, proliferating cell nuclear antigen; EdU, 5'-Ethynl-2'deoxyuridine.

\section{Discussion}

Although EM is not a malignant type of tumor, its pathological process, which is characterized by the infiltration, migration and invasion of uterine cells, is similar to that of cancer $(18,19)$. Therefore, regulating the proliferation, migration and invasion of uterine cells may be an important breakthrough in the treatment of EM.

BBR has a wide range of pharmacological activities, among which BBR exerts antitumor effects. Wang and Zhang (10) found that BBR suppressed the growth and metastasis of endometrial cancer cells via miR-101/COX-2. BBR and metformin 


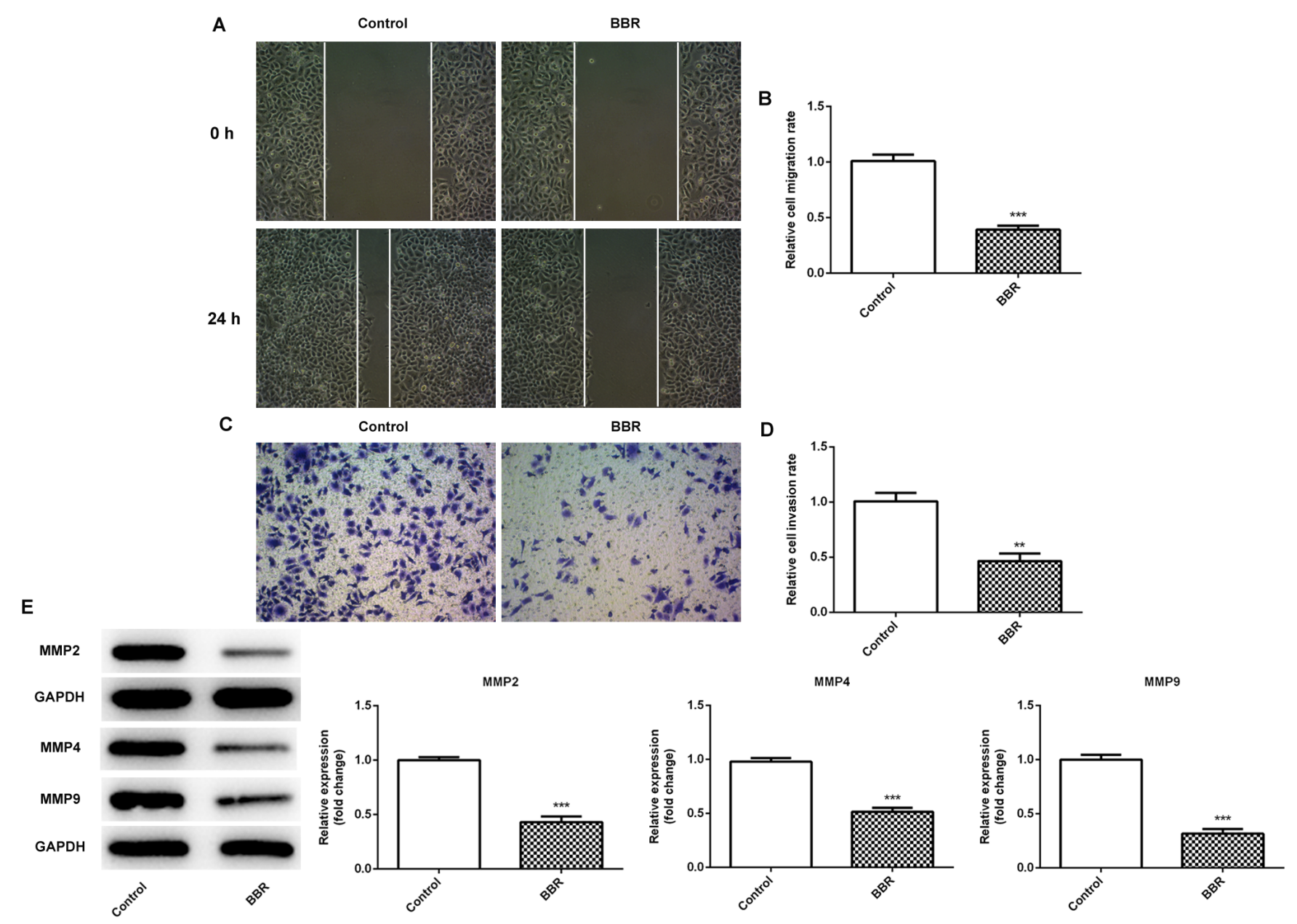

Figure 2. BBR inhibits the migration and invasion of human endometrial stromal cells. (A) A wound healing assay was used to measure cell migration. (B) Statistical analysis of the wound healing assay. (C) A Transwell assay was used to measure cell invasion. (D) Statistical analysis of the Transwell assay. (E) Protein expression of MMP2 and MMP9 was detected by western blotting. ${ }^{* *} \mathrm{P}<0.01,{ }^{* * * *} \mathrm{P}<0.001$ vs. Control. BBR, berberine; MMP, matrix metalloproteinase.
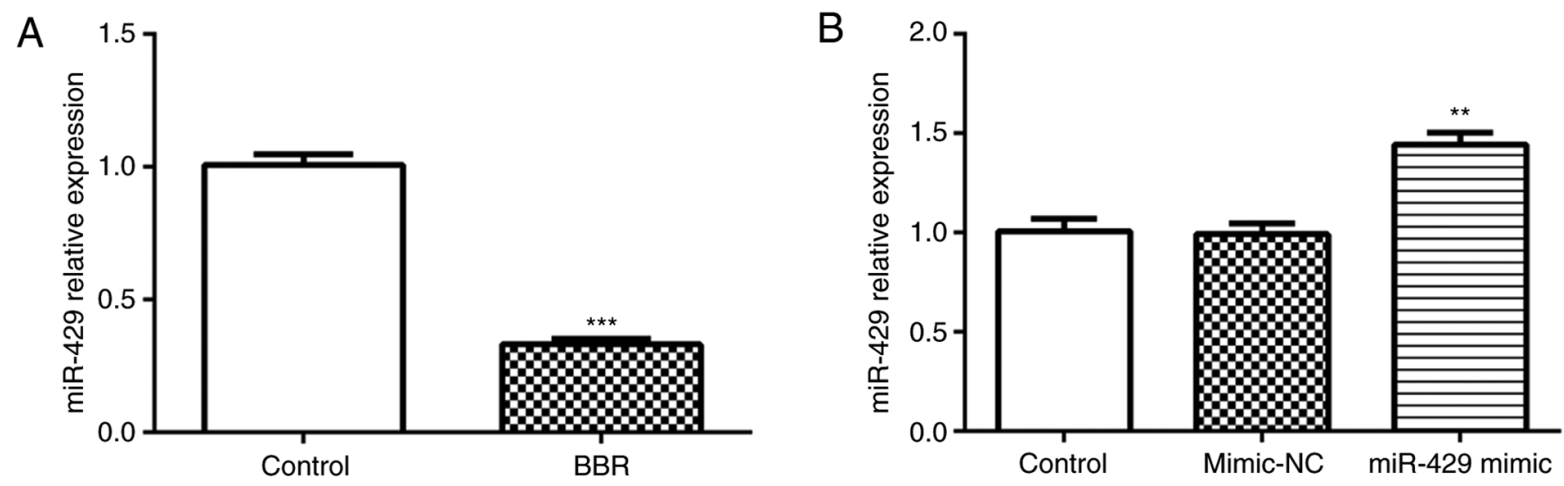

Figure 3. Expression of miR-429. (A) The expression of miR-429 was detected by RT-qPCR. ${ }^{* * *} \mathrm{P}<0.001$ vs. Control. (B) The expression of miR-429 following cell transfection was detected by RT-qPCR. ${ }^{* *} \mathrm{P}<0.01$ vs. mimic-NC. BBR, berberine; miR, microRNA; RT-qPCR, reverse transcription-quantitative PCR; NC, negative control.

have been revealed to prevent endometrial cancer cell migration and invasion by inhibiting the expression of lipolysis-stimulated lipoprotein receptor (20). Moreover, there are numerous links between endometrial cancer and EM (21-23). However, whether BBR exerts a therapeutic effect in EM was not reported in these previous studies. In the present study, the concentration of BBR used was $80 \mu \mathrm{M}$ and the incubation time was $24 \mathrm{~h}$. At present, the effect of this concentration has not been studied clinically, so the dose response experiment and further in vivo experiments need to be explored by our laboratory in future studies. It was found that BBR inhibited the proliferation, invasion and migration of HESCs.

It would be of interest to determine the underlying mechanisms of BBR in the regulation of proliferation, 


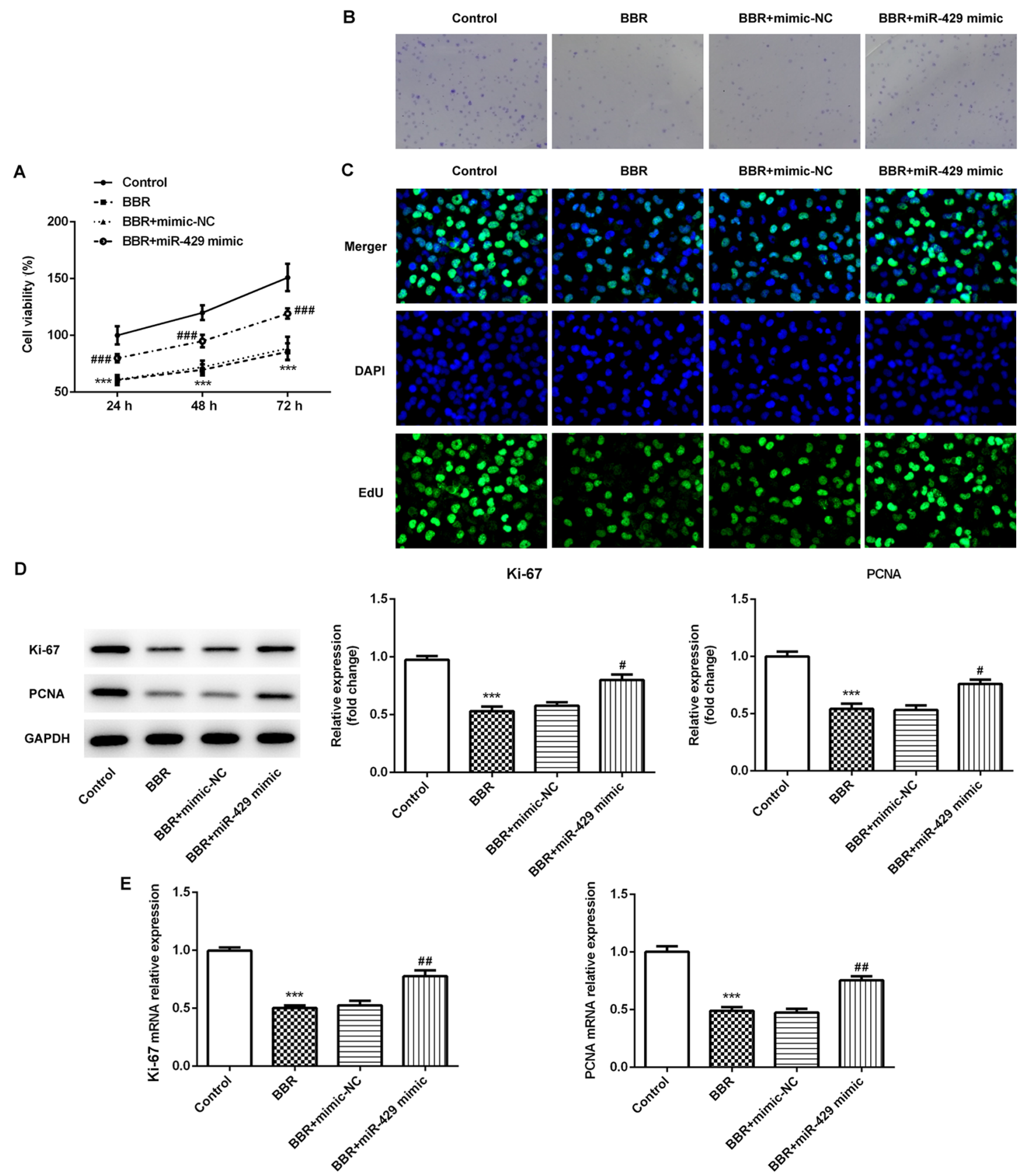

Figure 4. BBR inhibits the proliferation of human endometrial stromal cells by downregulating miR-429. (A) A Cell Counting Kit-8 assay was used to measure cell viability. (B) Cell proliferation was detected using a colony formation assay. (C) EdU assay was used to detect cell proliferation. (D) The protein expression levels of Ki-67 and PCNA were detected using western blotting. (E) The mRNA expression levels of Ki-67 and PCNA were detected by reverse transcription-quantitative PCR. ${ }^{* * *} \mathrm{P}<0.001$ vs. Control; ${ }^{\#} \mathrm{P}<0.05,{ }^{\# \#} \mathrm{P}<0.01,{ }^{\# \# \#} \mathrm{P}<0.001$ vs. BBR + mimic-NC. BBR, berberine; miR, microRNA; NC, negative control; Ki-67, proliferation marker protein Ki-67; PCNA, proliferating cell nuclear antigen.

invasion and migration of HESCs. The multiple pharmacological actions of BBR are the result of different molecular targets of this phytochemical. Certain studies have reported that BBR plays a therapeutic role in cancer by regulating the expression of miRNAs (24-26). In the present study, it was found that the expression of miR-429 decreased significantly following treatment of the cells with BBR. miR-429 has been reported to promote the progression of a variety of cancers. miR-429 has been demonstrated to competitively combine with long non-coding RNA SNHG22 to 

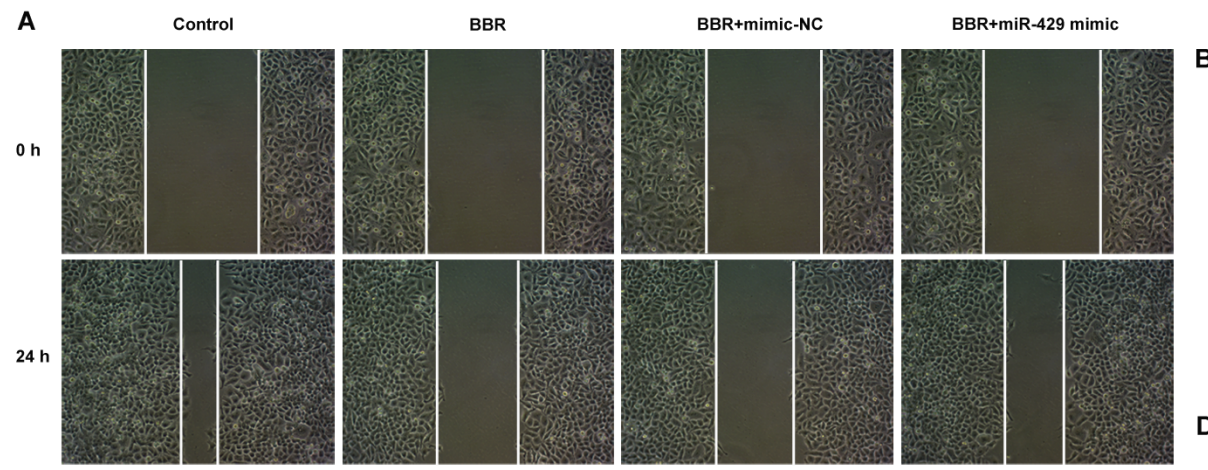

C Control BBR

$B B R+m i R-429$ mimic
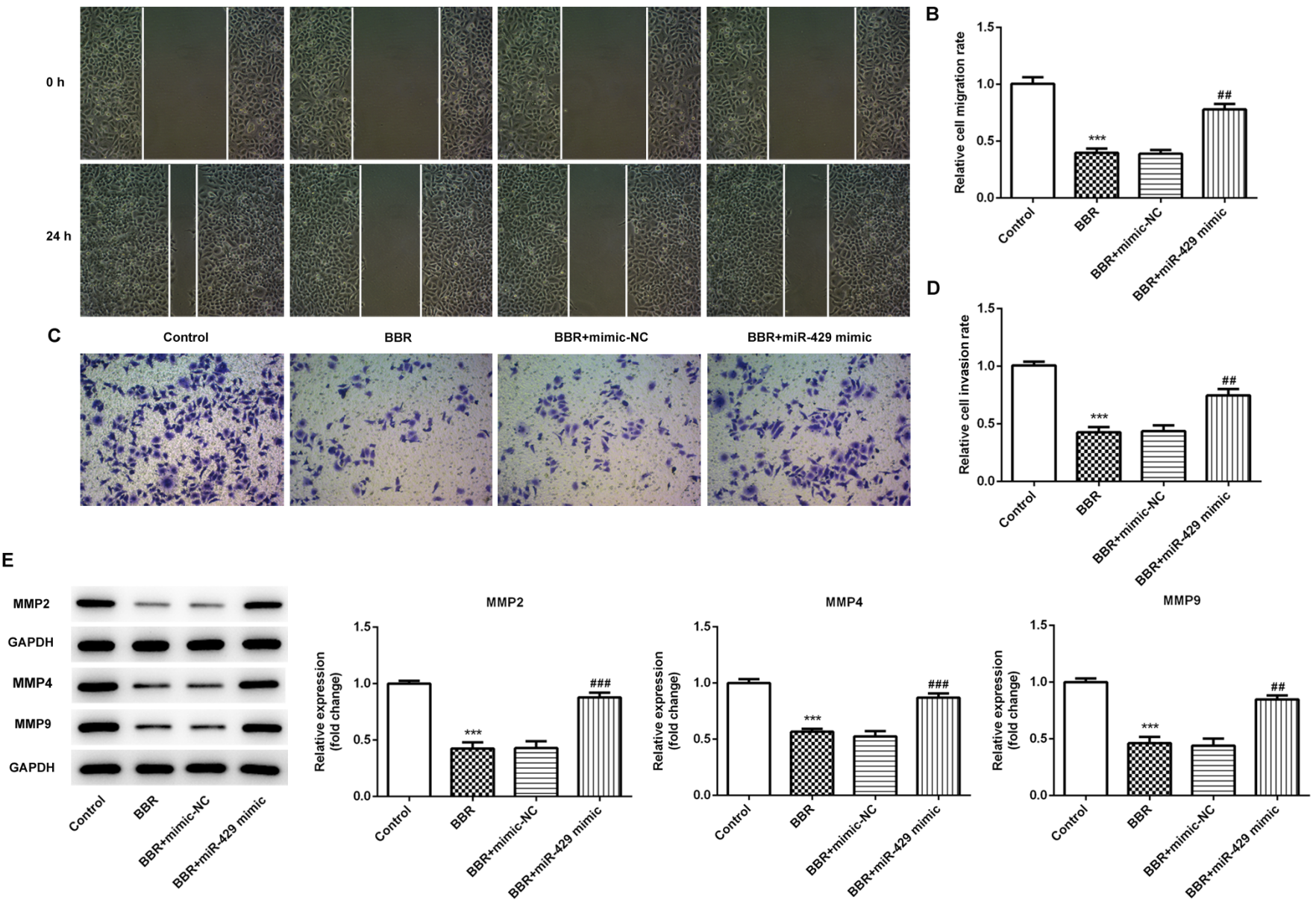

Figure 5. BBR inhibits the migration and invasion of human endometrial stromal cells by downregulating miR-429. (A) A wound healing assay was used to measure cell migration. (B) Statistical analysis of the wound healing assay. (C) A Transwell assay was used to measure cell invasion. (D) Statistical analysis of the Transwell assay. (E) The protein expression levels of MMP2 and MMP9 were detected by western blotting. ${ }^{* * *} \mathrm{P}<0.001$ vs. Control; ${ }^{\# \#} \mathrm{P}<0.01,{ }^{\# \# \#} \mathrm{P}<0.001$ vs. BBR + mimic-NC. BBR, berberine; MMP, matrix metalloproteinase; miR, microRNA; NC, negative control.

increase zinc finger E-box-binding homeobox 1 expression and promote the malignant development of thyroid papillary carcinoma (27). Increased proliferation and migration following the overexpression of miR-429 was previously observed in breast cancer cells, whereas the silencing of miR-429 attenuated tumor growth (28). Therefore, in the present study it was hypothesized that miR-429 plays a role in promoting cell proliferation, migration and invasion in EM. Following the overexpression of miR-429, it was found that the proliferative, invasive and migratory ability of HESCs were promoted, thus indicating that miR-429 successfully reversed the inhibitory effects of BBR on cell proliferation, invasion and migration. This indicated that BBR inhibited cell proliferation, migration and invasion by downregulating miR-429.

However, the present study only verified that BBR could inhibit the proliferation, invasion and migration of HESCs by regulating miR-429 at the cellular level. These findings were not validated in vivo or clinically, so it cannot be determined whether $80 \mu \mathrm{M}$ BBR is clinically applicable. Therefore, this study can only provide a theoretical basis for BBR in the clinical treatment of EM.

In conclusion, the present study demonstrated that BBR inhibited the proliferation, invasion and migration of HESCs by downregulating miR-429. The findings of the present study may provide the basis for the drug treatment of EM.

\section{Acknowledgements}

Not applicable.

\section{Funding}

Scientific and technological research project of education department of Jiangxi province (grant no. GJJ190640).

\section{Availability of data and materials}

The analyzed data sets generated during the present study are available from the corresponding author on reasonable request.

\section{Authors' contributions}

YG wrote the manuscript and analyzed the data. ZZ carried out the experiments, supervised the present study, searched the literature and revised the manuscript. YG and $\mathrm{ZZ}$ confirm the authenticity of all the raw data. Both authors read and approved the final manuscript. 


\section{Ethics approval and consent to participate}

Not applicable.

\section{Patients consent for publication}

Not applicable.

\section{Competing interests}

The authors declare that they have no competing interests.

\section{References}

1. Gordts S, Koninckx P and Brosens I: Pathogenesis of deep endometriosis. Fertil Steril 108: 872-885, 2017.

2. Czyzyk A, Podfigurna A, Szeliga A and Meczekalski B: Update on endometriosis pathogenesis. Minerva Ginecol 69: 447-461, 2017.

3. Li J, Ma J, Fei X, Zhang T, Zhou J and Lin J: Roles of cell migration and invasion mediated by twist in endometriosis. J Obstet Gynaecol Res 45: 1488-1496, 2019.

4. Kodaman PH: Current strategies for endometriosis management. Obstet Gynecol Clin North Am 42: 87-101, 2015.

5. Grimstad FW and Carey E: Periclitoral endometriosis: The dilemma of a chronic disease invading a rare location. J Minim Invasive Gynecol 22: 684-686, 2015.

6. Jin Y, Khadka DB and Cho WJ: Pharmacological effects of berberine and its derivatives: A patent update. Expert Opin Ther Pat 26: 229-243, 2016

7. Liu L, Fan J, Ai G, Liu J, Luo N, Li C and Cheng Z: Berberine in combination with cisplatin induces necroptosis and apoptosis in ovarian cancer cells. Biol Res 52: 37, 2019.

8. Du J, Sun Y, Lu YY, Lau E, Zhao M, Zhou QM and Su SB: Berberine and evodiamine act synergistically against human breast cancer MCF-7 cells by inducing cell cycle arrest and apoptosis. Anticancer Res 37: 6141-6151, 2017.

9. Abrams SL, Follo MY, Steelman LS, Lertpiriyapong K, Cocco L, Ratti S, Martelli AM, Candido S, Libra M, Murata RM, et al: Abilities of berberine and chemically modified berberines to inhibit proliferation of pancreatic cancer cells. Adv Biol Regul 71: 172-182, 2019.

10. Wang Y and Zhang S: Berberine suppresses growth and metastasis of endometrial cancer cells via miR-101/COX-2. Biomed Pharmacother 103: 1287-1293, 2018.

11. Jin P, Zhang C and Li N: Berberine exhibits antitumor effects in human ovarian cancer cells. Anticancer Agents Med Chem 15: 511-516, 2015.

12. Wu G, Zheng H, Xu J, Guo Y, Zheng G, Ma C, Hao S, Liu X, Chen H, Wei S, et al: miR-429 suppresses cell growth and induces apoptosis of human thyroid cancer cell by targeting ZEB1. Artif Cells Nanomed Biotechnol 47: 548-554, 2019.

13. Dai W, He J, Zheng L, Bi M, Hu F, Chen M, Niu H, Yang J, Luo Y, Tang W and Sheng M: miR-148b-3p, miR-190b, and miR-429 regulate cell progression and act as potential biomarkers for breast cancer. J Breast Cancer 22: 219-236, 2019.

14. Han Y, Zhao Q, Zhou J and Shi R: miR-429 mediates tumor growth and metastasis in colorectal cancer. Am J Cancer Res 7: $218-233,2017$
15. Liu H, Huang C, Wu L and Wen B: Effect of evodiamine and berberine on miR-429 as an oncogene in human colorectal cancer. Onco Targets Ther 9: 4121-4127, 2016.

16. Braicu OL, Budisan L, Buiga R, Jurj A, Achimas-Cadariu P, Pop LA, Braicu C, Irimie A and Berindan-Neagoe I: miRNA expression profiling in formalin-fixed paraffin-embedded endometriosis and ovarian cancer samples. Onco Targets Ther 10: 4225-4238, 2017.

17. Livak KJ and Schmittgen TD: Analysis of relative gene expression data using real-time quantitative PCR and the 2(-Delta Delta C(T)) method. Methods 25: 402-408, 2001.

18. Meng X, Liu J, Wang H, Chen P and Wang D: MicroRNA-126-5p downregulates BCAR3 expression to promote cell migration and invasion in endometriosis. Mol Cell Endocrinol 494: 110486 , 2019.

19. Garcia-Solares J, Dolmans MM, Squifflet JL, Donnez J and Donnez O: Invasion of human deep nodular endometriotic lesions is associated with collective cell migration and nerve development. Fertil Steril 110: 1318-1327, 2018

20. Shimada H, Satohisa S, Kohno T, Takahashi S, Hatakeyama T, Konno T, Tsujiwaki M, Saito T and Kojima T: The roles of tricellular tight junction protein lipolysis-stimulated lipoprotein receptor in malignancy of human endometrial cancer cells. Oncotarget 7: 27735-27752, 2016.

21. Kim JJ, Kurita T and Bulun SE: Progesterone action in endometrial cancer, endometriosis, uterine fibroids, and breast cancer. Endocr Rev 34: 130-162, 2013.

22. Chen JJ, Xiao ZJ, Meng X, Wang Y, Yu MK, Huang WQ, Sun X, Chen H, Duan YG, Jiang X, et al: MRP4 sustains Wnt/ $\beta$-catenin signaling for pregnancy, endometriosis and endometrial cancer. Theranostics 9: 5049-5064, 2019.

23. Kajiyama H, Suzuki S, Yoshihara M, Tamauchi S, Yoshikawa N, Niimi K, Shibata K and Kikkawa F: Endometriosis and cancer. Free Radic Biol Med 133: 186-192, 2019.

24. McCubrey JA, Lertpiriyapong K, Steelman LS, Abrams SL, Yang LV, Murata RM, Rosalen PL, Scalisi A, Neri LM, Cocco L, et al: Effects of resveratrol, curcumin, berberine and other nutraceuticals on aging, cancer development, cancer stem cells and microRNAs. Aging (Albany NY) 9: 1477-1536, 2017.

25. Dai W, Mu L, Cui Y, Li Y, Chen P, Xie H and Wang X: Berberine promotes apoptosis of colorectal cancer via regulation of the long non-coding RNA (lncRNA) cancer susceptibility candidate 2 (CASC2)/AU-binding factor 1 (AUF1)/B-cell CLL/lymphoma 2 (Bcl-2) axis. Med Sci Monit 25: 730-738, 2019.

26. Lo SN, Wang CW, Chen YS, Huang CC, Wu TS, Li LA, Lee IJ and Ueng YF: Berberine activates aryl hydrocarbon receptor but suppresses CYP1A1 induction through miR-21-3p stimulation in MCF-7 breast cancer cells. Molecules 22: 1847, 2017.

27. Gao H, Sun X, Wang $\mathrm{H}$ and Zheng Y: Long noncoding RNA SNHG22 increases ZEB1 expression via competitive binding with microRNA-429 to promote the malignant development of papillary thyroid cancer. Cell Cycle 19: 1186-1199, 2020.

28. Cava C, Novello C, Martelli C, Lodico A, Ottobrini L, Piccotti F, Truffi M, Corsi F, Bertoli G and Castiglioni I: Theranostic application of miR-429 in HER2+ breast cancer. Theranostics 10 50-61, 2020.

(i) $(-)$ This work is licensed under a Creative Commons Attribution-NonCommercial-NoDerivatives 4.0 International (CC BY-NC-ND 4.0) License. 